\title{
LA FANTASÍA DEBE SER DEVUELTA A LA ARQUITECTURA
}

\author{
Ana Tostões
}

\begin{abstract}
El arquitecto luso-africano Pancho Guedes (1925), fue uno de los protagonistas principales de la revalorización de la arquitectura moderna en Mozambique (colonia portuguesa hasta su independencia en 1975). A través de sus escritos y su producción arquitectónica conectó diferentes disciplinas y culturas, estableciendo afinidades con diversos artistas como el pintor Malangatana Ngwenya (1936-2009). Su "fantástica y mágica arquitectura" es el resultado de la recepción de estímulos provenientes de una enorme red mundial de artistas y pensadores que recopiló de fuentes como: el propio Movimento Moderno, desde la Sudáfrica de Martiessen hasta el Brasil de Lucio Costa y Óscar Niemeyer; la contestación crítica a los CIAM por parte del Team 10, del cual fue fundador en el congreso de Royaumont junto con los Smithson, Aldo Van Eyck, Candilis y Giancarlo di Carlo; y, finalmente, un gran número de artistas africanos a los que impulsó.
\end{abstract}

Palabras clave: Arte, arquitectura, África, movimiento moderno

Keywords: Art, Architecture, Africa, Modern Movement

Pancho Guedes ${ }^{1}$ (1925-2015) fue un arquitecto luso-africano (Universidad de Witwatersrand, 1953) que contribuyó notablemente a reconsiderar la modernidad arquitectónica mediante sus escritos y su labor proyectual, apoyado en el contacto con diferentes disciplinas y culturas y en su relación con diversos creadores, especialmente con el pintor Malangatana Ngwenya (1936-2009). Aunque ejerció en Mozambique, colonia portuguesa hasta su independencia en 1975, su arquitectura misteriosa y fantástica es producto del estímulo de una amplia red, que él mismo promovió, de artistas y pensadores de distintos países y de muy diversos orígenes, tanto nuevos artistas africanos que encontraron apoyo en Guedes como arquitectos adscritos al movimiento moderno, concretamente colaboradores sudafricanos, entre los que cabe destacar a Martienssen. Por otra parte, contó con la estimulante influencia brasileña personificada en Lúcio Costa y Oscar Niemeyer; el reflexivo legado de Frank Lloyd Wright o el movimiento de discusión crítica de los CIAM al amparo del Team X, al que se había unido como miembro fundador en el encuentro de Royaumont junto a los Smithson, Aldo Van Eyck, Candilis y Giancarlo di Carlo.

Además de ser uno de los más célebres arquitectos de Mozambique, Pancho tenía habilidad para detectar el talento y favorecer la creatividad, lo que le sirvió para establecer una red de artífices en la que podría decirse que él actuaba de mediador entre el arte y la arquitectura $^{2}$.

En Lourenço Marques (actual Maputo), surgió una profunda complicidad entre Pancho y Malangatana, el pintor surrealista cuya capacidad de invención no conocía límites ${ }^{3}$. Atraído por lo sobrenatural, Malangatana azuzaba en Pancho el deseo de "oír las voces que hablan desde el otro lado del sueño"4. Durante los años cincuenta, en el África del apartheid entre Mozambique, Rodesia y Sudáfrica, supo ver la necesidad de instaurar un arte auténtico y genuino, el arte de los verdaderos artistas ${ }^{5}$, así que exploró una arquitectura rebosante de significado, portadora de una dimensión personal basada en la investigación de todas las dimensiones formales y en el potencial que tienen los elementos arquitectónicos para contener y expresar emociones: "Reivindico que los arquitectos tengan los mismos derechos y la misma libertad que han tenido los pintores y los poetas desde hace tanto tiempo"6. Pancho quería hacerse con los elementos temáticos universales de las culturas primitivas y mezclarlos con su propia cultura arquitectónica, más sofisticada, para conseguir recrear en sus edificios la atmósfera de las pinturas de Giorgio de Chirico. Pancho sabía que la arquitectura no se percibe como una experiencia intelectual, sino como una sensación, una emoción ${ }^{7}$, por lo que perseguía esa cualidad "desaparecida de entre los arquitectos hace mucho tiempo, pero que puede materializarse en una arquitectura espontánea capaz de producir una intensidad mágica"8.

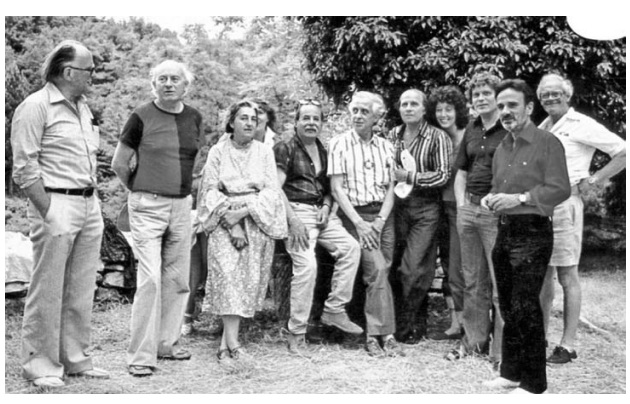

Fig. 1. Team X, Bonnieux, France, 1977. ( ) En Pedro Guedes (ed.), Pancho Guedes. Vitruvius Mozambicanus, Lisboa, Museo Coleção Berardo, 2009.

1. El nombre completo de Pancho Guedes era Amâncio d'Alpoim Miranda Guedes; pero adquiere varias formas: Amâncio Guedes 0 Pancho Guedes, A. Miranda Guedes, A. de Alpoim Guedes, Amâncio D’Alpoim Guedes, Amâncio de Miranda Guedes.

2. NGWENYA, Malangatana, "Pancho Guedes visto por Malangatana", en Savana, 5 de marzo de 2010. POMAR, Alexandre (ed.), The Africas of Pancho Guedes, the Dori and Amâncio Guedes Collection, CML-Sextante, Lisboa, 2010, p. 56.

3. GUEDES, Dorothy, "Vinte e Quatro Poemas de Malangatana", en NGWENYA, Malangatana, Vinte e Quatro Poemas, ISPA, Lisboa, 1996, p. 7

4. GUEDES, Pancho, Manifestos, Papers, Lectures, Publications, OA, Lisboa, 2007, p. 55

5. GUEDES, Amâncio, "Mr. Tito Zungu. Master of the Decorated Envelope". Ibid. p. 111

6. GUEDES, Amâncio, "A Wrightian Thesis from the Early Fifties". Ibid. p. 12.

7. HUET, Bernard, introducción a GUEDES, Amâncio, "Y aura-t-il une architecture?", en L'Architecture d'Aujourd'hui, n. 102, 1962, p. 42.

8. GUEDES, Amâncio, "A Wrightian Thesis from the Early Fifties", op. cit. 
9. Como declara Pancho, "para algunos, el movimiento moderno ha cumplido sus objetivos y la arquitectura ha entrado en una época de refinamiento y clasicismo. Verdaderamente, el cáncer de los estilos vuelve a cernirse sobre nosotros más mortífero y amenazante que nunca. Otros -nosotros, los que día a día miramos de frente a la soledad- sabemos que seremos unos proscritos hasta el fin de nuestros días a no ser que nos traicionemos a nosotros mismos". GUEDES, Amâncio, "Y aura-t-il une architecture?", en L'Architecture d'Aujourd'hui, n. 102, 1962, pp. 42-48.

10. Pancho se casó con Dorothy Ann Philips (Dori) en 1947 11. POMAR, Alexandre (ed.), The Africas of Pancho Guedes, the Dori and Amâncio Guedes Collection, op. cit., p. 8.

12. GUEDES, Pedro, Vitruvius Mozambicanus, Berardo Collection Museum, Lisboa, 2009, p. 165.

13. TZARA, Tristan, "Introduction to Guedes' Lecture", de GUEDES, A. D'Alpoim, "Things Are Not What They Seemed to Be", actas del Primer Congreso Internacional sobre la Cultura Africana celebrado en la National Gallery, Salisbury, Rodesia, 1-11 de agosto de 1962

14. "Visionary Architecture", exposición del MoMA en 1960 RUDOFSKY, Bernard (1905 - 1988), Architecture Without Architects: A Short Introduction to Non-Pedigreed Architecture Doubleday, New York, 1964, traducido al español por Raú Grego como Arquitectura sin arquitectos: breve introducción a la arquitectura sin genealogía, Editorial Universitaria, Buenos Aires, 1973: "La historia de la arquitectura, tal como se ha escrito y enseñado en Occidente, nunca se ha preocupado más que por unas pocas culturas selectas".

15. ROGERS, Ernesto Nathan, "Continuità o Crisi”, en Casa bella-Continuità, n. 215, 1957.

16. GIEDION, Sigfried, Space, Time and Architecture. The Growth of a New Tradition, Harvard University Press, Cambridge (Massachusetts), 1941, traducido al español por Jorge Sainz como Espacio, tiempo y arquitectura. Origen y desarrollo de una nueva tradición, Reverté, Barcelona, 2009.

17. Se trataba de un país imperialista peculiar en el sentido de que en el siglo XVIII los poderes políticos portugueses se habían planteado transferir la capitalidad de Lisboa a Río de Janeiro, en Brasil, su colonia americana.

18. GUEDES, Amâncio d'Alpoim, "Lembrança do pinto Malangatana Valente Ngwenya quando ainda jovem", en NAVARRO, Júlio, Malangatana Valente Ngwenya, Caminho, Lisboa, 1998, p. 9.

19. POMAR, Alexandre, "There were a lot of people hovering around", en POMAR, Alexandre (ed.), The Africas of Pancho Guedes, the Dori and Amâncio Guedes Collection, op. cit., p. 40 20. GUEDES, Amâncio d'Alpoim, "Lembrança do pintor Malangatana Valente Ngwenya quando ainda jovem", en NAVARR0, Júlio, Malangatana Valente Ngwenya, op. cit, p. 9.

21. SANTIAGO, Miguel, Pancho Guedes - Metamorfoses Espaciais, Caleidoscópio, Lisboa, 2007.

22. BEINART, Julian, "Amâncio Guedes, architect of Lourenço Marques", en Architectural Review, n. 770, abril de 1961, pp. 240-251; RICHARDS, James Maude, "Emergence of a new and original figure: remarkable work by Amâncio Guedes", en The Times, 17 de mayo de 1961. Véase MARTINS, João Paulo, "A Difícil Internacionalização", en TOS TÕ̃E, Ana (ed.), Arquitectura Moderna Portuguesa, IPPAR Lisboa, 2004, p. 166.

23. Declaración personal, 1 de noviembre de 2010 en POMAR, Alexandre, "There were a lot of people hoverin around", en POMAR, Alexandre (ed.), The Africas of Panch Guedes, the Dori and Amâncio Guedes Collection, op. cit. p. 54; GUEDES Pedro (ed.), Vitruvius Mozambicanus, op. cit.
En los años cincuenta, esta búsqueda fue el resultado del deseo de crear una modernidad alternativa, diferente al mecánico estilo internacional que también se estaba abriendo paso en Áfri$\mathrm{ca}^{9}$. A diferencia de la mayoría de los arquitectos que trabajaban en este continente, que se veían forzados a entablar en sus dibujos un diálogo con los condicionantes climáticos, Pancho asumió para el creador el derecho a la inocencia, avivado por la sensualidad y el dramatismo de la cultura africana circundante. La creación y engrosamiento de la colección de obras de arte africano de Dori ${ }^{10}$ y Pancho, que recientemente ha sido expuesta en Lisboa, da testimonio de su continuado interés por conversar con otras formas de expresión ${ }^{11}$. Como él mismo declaró, estos objetos que fue recopilando le ayudaron a liberarse "del predominante punto de vista eurocéntrico del hombre blanco que vive en tierra de otros"12. El afán por descubrir una modernidad alternativa respondía tanto a una necesidad interior como a un continente que despertaba a la contemporaneidad, a un nuevo mundo en plena efervescencia ${ }^{13}$. Pancho es testigo y parte activa de un tiempo en el que la arquitectura está abierta a la cultura popular, en el que se aceptan la arquitectura sin arquitectos y la arquitectura de la fantasía ${ }^{14}$. Pero se trata igualmente de un tiempo complejo en el que la continuidad o $\mathrm{crisis}^{15}$ del movimiento moderno dio pie a múltiples soluciones, que Giedion identificó como el resultado de la ecuación equivalente entre razón y emoción ${ }^{16}$.

Pancho reunía las condiciones para seguir un camino propio, original, alternativo e idiosincrático. Además de su gran talento, su vasta cultura y una curiosidad genuina y proclive a la experimentación, las circunstancias habían querido que viviera en África en ese momento y supo aprovecharlo con habilidad. Por una parte, se encontraba al margen de la cultura dominante europea y eurocéntrica, en una colonia africana de un país imperialista europeo bastante peculiar ${ }^{17}$; por otra parte, le había tocado vivir el periodo de emancipación colonial que se expandía por toda África, donde, a pesar de la presencia imperialista, "todo parecía posible"18.

Como indica Pomar, Pancho "era uno de los arquitectos más innovadores de los que estaban construyendo en África, así como uno de los tres o cuatro intermediarios blancos de renombre que buscaban y auspiciaban el arte africano contemporáneo en distintos puntos del continente. Lourenço Marques era en aquel tiempo (en los primeros años sesenta y antes de la oleada de detenciones que siguieron al inicio de la guerra de la independencia), una ciudad dinámica y una de las capitales culturales de África, debido, en gran medida, a los contactos internacionales de Pancho en las disciplinas con mayor capacidad de comunicación con el mundo exterior, como la arquitectura y las artes visuales" 19 .

\section{ERA UN MUNDO EN EL QUE TODO PARECÍA POSIBLE}

Desde Lourenço Marques, Pancho teje una "red de afinidades" que pone en contacto a creadores africanos, americanos y europeos, lo que, en el marco de la dictadura colonial del régimen portugués, le permite tener la audacia de postularse como representante mozambiqueño en la Bienal Internacional de Arte de São Paulo de 1961. Él mismo declara: "Durante los años cincuenta y sesenta había algo incesante y extraordinario en la hermosa ciudad que habían construido los portugueses en menos de medio siglo y a la que llamaron Lourenço Marques. [...] En aquella época, Mozambique era un mundo aislado e irreal en el que desde el imperio solo nos llegaban buenas noticias, inauguraciones y discursos. Era un mundo de rumores, de secretos, de confidencias y de una creciente maraña de soplones y agentes, pero en el que, a pesar de eso, todo parecía posible"20.

1960 es el año de los descubrimientos, en el que tiene lugar la gran gira europea "annus mirabilis MCMLX"21, en la que Pancho se encuentra con Alison y Peter Smithson en Londres, visita los edificios de Távora y Siza en Oporto, conoce en la capital británica a los editores de Architectural Design y prepara su debut internacional en las revistas de mayor prestigio: en 1961 el arquitecto sudafricano Julian Beinart publica el trabajo de Pancho en Architectural Review, y poco antes se había reunido con Reyner Banham y James Maude Richards, que también escribió sobre él en The Times ${ }^{22}$. Pancho reconoce que durante los primeros años sesenta "deambulaba por ahí mucha más gente, había muchos pintores"23; especialmente por su estudio, un taller de incesante actividad y ambiente distendido en el que se reunían artistas y artesanos locales. Frank McEwen, Ulli Beier, Julian Beinart y Pancho Guedes son las figuras clave de una red africana de mecenazgo y patrocinio del arte muy activa en los tiempos de la independencia de los años sesenta. 


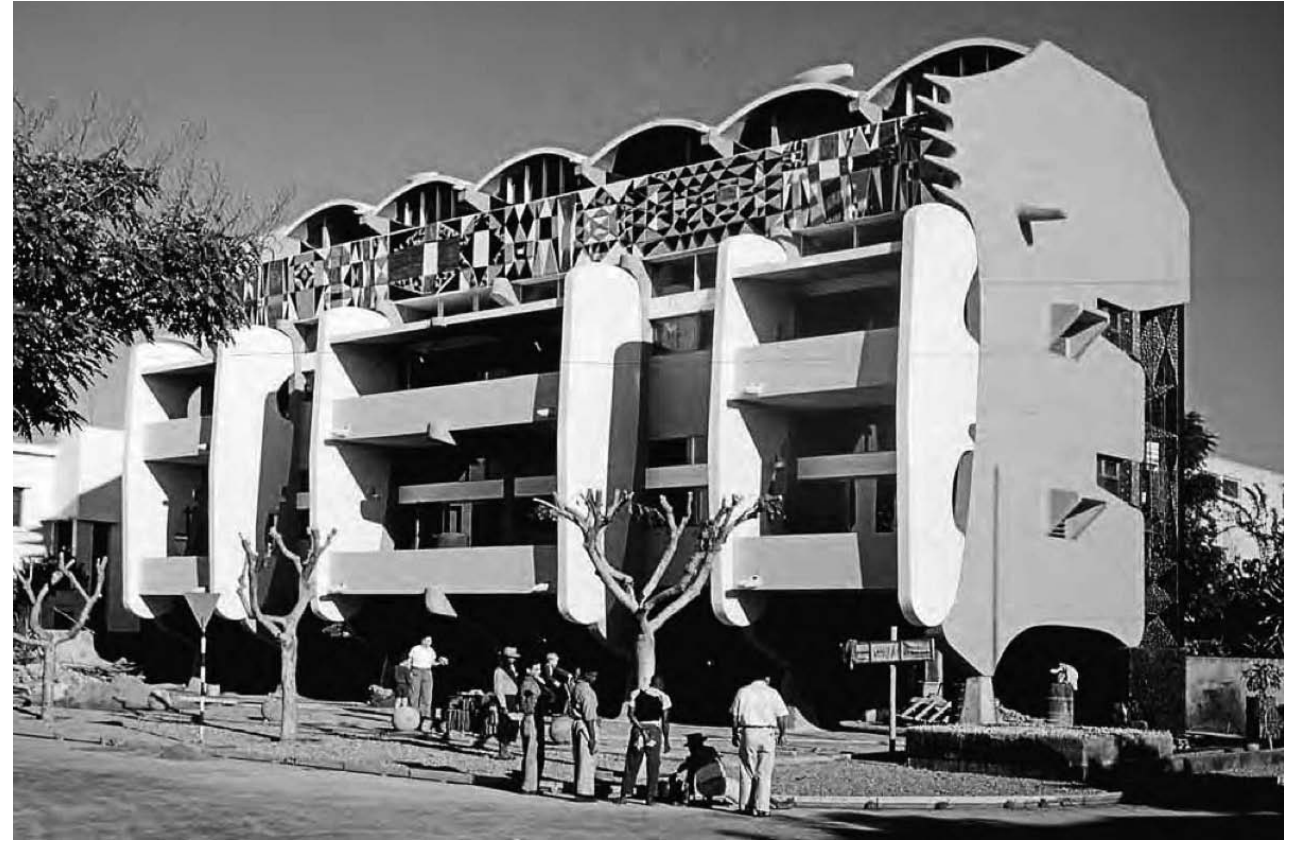

2

La década se inició con la inauguración del Summer Workshop en enero de 1961. En un comienzo no se pensaba dar formación a artistas negros. De hecho, se había concebido como un curso de verano para estudiantes de la Universidad de Witwatersrand. Sin embargo, los trabajadores que aún se encontraban finalizando las obras del edificio de la Escuela Infantil Piramidal, donde tenían lugar las sesiones, se unieron al grupo y ofrecieron sus propias versiones de los temas, participando con materiales no académicos ${ }^{24}$. En palabras de Pancho Guedes, "llegaron e hicieron por sí mismos unos dibujos maravillosos, mucho mejores que los de nuestros estudiantes" 25 . Pocos meses después tuvo lugar la primera exposición individual de Malangatana en Lourenço Marques y un segundo taller en Ibadán para profesores de arte nigerianos, coordinado por Julian Beinart y Pancho Guedes.

Finalmente, entre septiembre y diciembre, la inaudita presencia de Pancho en la bienal de São Paulo como único representante de un país llamado Mozambique, que el Centro de Información y Turismo nacional hizo oficial con la presentación del edificio del O Leão Que Ri (El León que Ríe), le abrió definitivamente las puertas del ámbito internacional. Al año siguiente recibe la invitación para formar parte del Team X y participa en el encuentro de la abadía de Royaumont. También en 1962 le llega la popularidad en Francia gracias a L'Architecture d'Aujourd'hui, con un artículo titulado "Y aura-t-il une architecture?” (¿Habrá una arquitectura?), que se incluye en el número que Bernard Huet dedica a las "Fantastic Architectures" ${ }^{26}$ en la línea de la exposición "Visionary Architecture" que el MoMA había organizado en $1960^{27}$. A estas apariciones públicas les siguió la colaboración en World Architecture, editada en Londres por John Donat y en cuyos números de 1964, 1965 y 1967 aparece A. D. A. Guedes como "editor adjunto de Mozambique".

En el circuito internacional, Pancho logró el reconocimiento simultáneamente como arquitecto, mecenas y difusor del arte africano. La publicación en París de su artículo sobre las casas pintadas de la comunidad sudafricana de los Ndebele, titulado "Les Mapogga", fue portada de la revista dirigida por André Bloc Aujourd'hui: Art et Architecture ${ }^{28}$ y supuso toda una revolución por tratarse de "el primero en poner de relieve el formalismo arquitectónico y escultórico de las viviendas"29. El mecenazgo de Malangatana había comenzado en 1959, el "taller de verano" de Lourenço Marques tiene lugar en 1961, el análisis crítico de su obra se produce al año siguiente con un artículo de Julian Beinart en la revista Black Orpheus, que había fundado Ulli Beier en Ibadán. En 1962, el acontecimiento más importante fue el Primer Congreso Internacional de Cultura Africana, organizado por Frank McEwen (1907-1994) $)^{30}$ para profundizar en la estética del arte africano contemporáneo y que tuvo lugar en la National Gallery de Salisbury, Rodesia (hoy Harare, Zimbabue), entre el uno y el once de agosto.

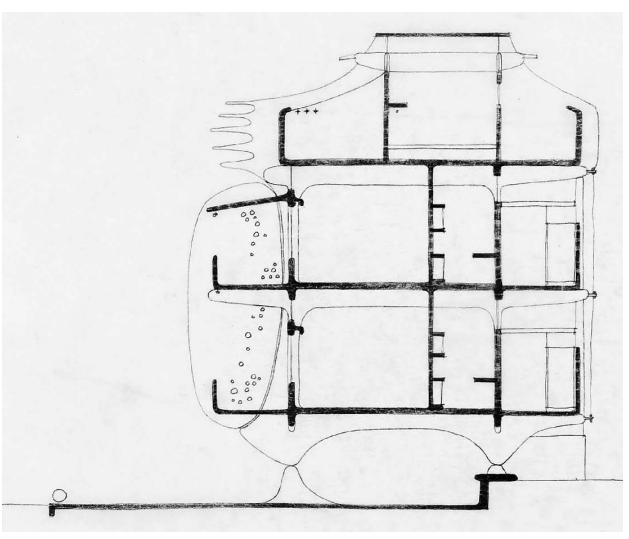

3

Fig. 2. El edificio 0 Leão Que Ri (El León que Ríe) durante su finalización (“stiloguedes”), Lourenço Marques (Maputo), 1958 (archivo de P. G.)

Fig. 3. Pancho Guedes, El edificio 0 Leão Que Ri (El León que Ríe), Lourenço Marques (Maputo), Mozambique, 1954-55. Sección, lápiz sobre papel, $30 \times 50 \mathrm{~cm}$. (C) En Pedro Guedes (ed.), Pancho Guedes. Vitruvius Mozambicanus, Lisboa, Museu Coleção Berardo, 2009.

24. BEINART, Julian, "Visual Education for Emerging Cultures: The African Opportunity", en KEPES, Gyorgy, The Education of Vision, Baziller, Nueva York, 1965, traducido al español como La educación visual, Navarro, México, 1968.

25. GUEDES, Pancho y BEIER, Ulli, "Interview, Johannesburg, September 19th 1980", en GUEDES, Pedro (ed.) Vitruvius Mozambicanus, op. cit., p. 27.

26. GUEDES, Amâncio "Y aura-t-il une architecture?", en L'Architecture d'Aujourd'hui, n. 102, 1962, pp. 42-48. El número "Fantastic Architectures" incluía tanto a los que iban por libre, como Facteur Cheval, Gaudí, Ios innovadores modernos, Mendelsohn, Le Corbusier y Wright como a los visionarios Pascal Hauserman, Bruce Goff y Paolo Soleri.

27. Su éxito internacional obtuvo una discreta mención en la revista Arquitectura (n. 79, julio de 1963, Lisboa): "Miranda Guedes, arquitecto de Lourenço Marques".

28. GUEDES, A. de Alpoim, "Les Mapogga”, en Aujourd'hui: Art et Architecture, n. 37, junio de 1962, pp. 58-65.

29. FONTANA ANTONELLI Giovanni, "Inventer une nouvelle illusion: le cas renommé des Southern Ndebele", extraído de http://www.international.icomos.org/victoriafalls2003/ papers.htm

30. Frank McEwen (1907-1994), artista británico muy activo entre los vanguardistas parisinos de la década de 1930. Más tarde llegó a ser el delegado en París del British Council y primer director de la Rhodes National Gallery de Rodesia (hoy Zimbabue) entre 1956 y 1973, donde fundó la Workshop School e impulsó el movimiento escultórico Shona. En POMAR, Alexandre (ed.), The Africas of Pancho Guedes, the Dori and Amâncio Guedes Collection, op. cit. 


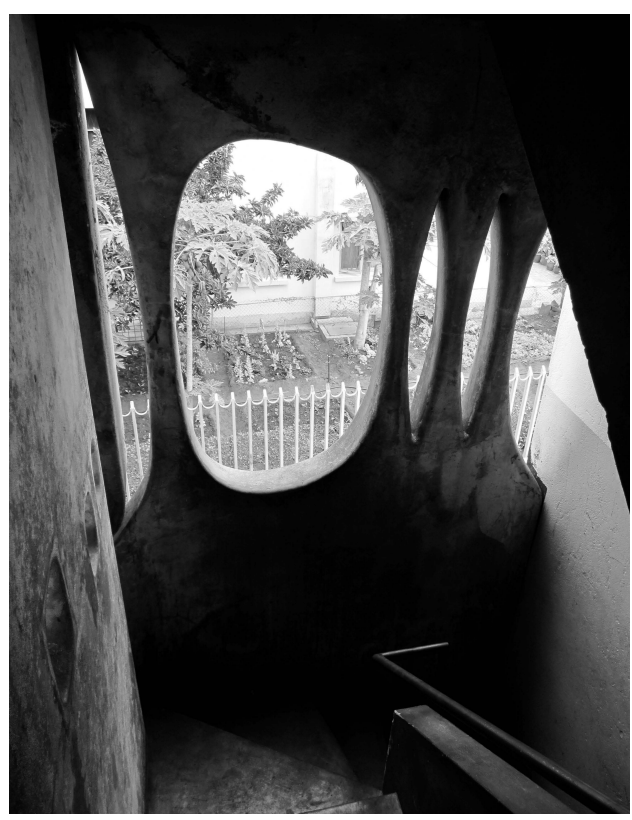

Fig. 4. Pancho Guedes, El edificio 0 Leão Que Ri (El León que Ríe), Lourenço Marques (Maputo), Mozambique, 1954-55. (C) Ana Tostões.
El congreso puso de manifiesto el creciente interés por la cultura africana y contó con la participación de treinta y siete delegados, entre los que se encontraba Alfred Barr, del MoMA de Nueva York; William Fagg del Museo Británico; Jean Laude de La Sorbona; el pintor surrealista y presidente del Institute of Contemporary Art (ICA) de Londres, Roland Penrose, acompañado por el fotógrafo Lee Miller; James Porter de la Howard University de Washington; Udo Kultermann, investigador y autor de varios libros sobre arquitectura moderna africana; el poeta dadaísta Tristan Tzara; John Russel, que en ese momento era crítico en The Sunday Times ${ }^{31}$; el musicólogo sudafricano Hugh Tracey; y el historiador nigeriano Saburi O. Biobaku, vicerrector de la Universidad de Ife, quien inauguró el congreso con un mensaje de apoyo desde Nigeria ${ }^{32}$.

En su discurso sobre el arte contemporáneo africano, Frank McEwen se cuestionaba: ¿qué le estaba ocurriendo al arte tradicional africano en ese momento en que debía enfrentarse a la evolución de las antiguas estructuras sociales? ¿Qué le depararía el futuro al arte africano? Eran ya evidentes las amenazas a las que McEwen denominaba "artes de aeropuerto", y Pancho dio a conocer, además de a Malangatana, la obra de otros cuatro artistas mozambiqueños en la exposición dedicada al arte africano no tradicional ${ }^{33}$.

Presentado como arquitecto e impulsor del arte contemporáneo africano, Pancho Guedes fue uno de los oradores de la sesión inaugural. Su ponencia sobre su propia obra como arquitecto y artista establecido en África, titulada "Things are not what they seem to be - the auto-biofarcical hour" 34 (Las cosas no son lo que parecen: la hora de la autobiografarsa), contó con la introducción y la complicidad del dadaísta Tzara. Pat Pearce, artista de Rodesia y colaborador de Frank McEwen, recordaba que "uno de los momentos más interesantes del congreso se produjo con la aparición de Pancho Guedes". En palabras de John Russell, "puso a todo el auditorio en pie con un poético y deslumbrante alegato sobre cómo la fantasía debe ser devuelta a la arquitectura. Sentí que Pancho había atrapado la misma esencia de la cultura africana que Picasso hacía unos años, solo que captando mucho más: la simplicidad, la fantasía y el humor entrañables que forman parte importante de la vida y del arte africanos" ${ }^{\prime 35}$.

La exposición que se había montado en paralelo al congreso fue una enorme muestra de arte africano, la mayor de todos los tiempos, según la revista Time ${ }^{36}$. Pomar cuenta que se iniciaba con las culturas previas a la llegada de las influencias europeas y continuaba hasta "el arte africano no tradicional de los años sesenta". En esa época, mientras que "para la mayor parte de los colonizadores blancos el arte africano siempre había sido pura incoherencia, prueba de que los nativos africanos no poseían instinto cultural" (el Rhodesia Herald dijo haber visto "únicamente crudeza, primitivismo y barbarie"), McEwen quería demostrar que "el movimiento moderno occidental en su conjunto está en deuda con el África primitiva. [...] Es un hecho que hay pocos artistas de estilo contemporáneo que carezcan por completo de influencias africanas, bien asimiladas pero evidentes"37. Por aquel entonces, Barr adquirió las primeras obras de arte moderno africano para el MoMA. Al año siguiente, en 1963, Pancho adoptó públicamente una postura crítica frente al planeamiento colonial poniendo en tela de juicio las normativas en un artículo titulado "La ciudad enferma" que publicó A Tribuna en Lourenço Marques ${ }^{38}$. Como cuenta Malangatana, "para Pancho era muy doloroso ver el contraste entre el estado ruinoso de la "ciudad de cañizo', carente de higiene y de los servicios más básicos, y la 'ciudad de cemento', situadas una junto a la otra. En los sesenta escribió un largo artículo sobre esto, con el objetivo de atraer la atención del gobierno colonial hacia estos problemas, y fue muy atacado tras su publicación. Incluso el Ayuntamiento reaccionó en su contra. Pero no le importó. Quería encontrar una solución que al menos aportara alguna medida de alivio, por pequeña que fuera, para aquellos que vivían en tan abyecta pobreza. La burguesía portuguesa se alineó con la postura oficial y también mostró su desaprobación, pero la población de las zonas más degradadas recibió a Pancho con gran alborozo, y aún hoy lo recuerdan. [...] Sin la cautivadora mano del arquitecto Miranda Guedes yo no habría tenido la luz de referencia que ha iluminado el tortuoso sendero de la vida" ${ }^{" 39}$.

\section{LOS EDIFICIOS DEBERÍAN SONREÍR Y HABLAR}

La identificación de Lourenço Marques como uno de los focos de un África en evolución, a pesar de la huella colonialista (en una época caracterizada por la "euforia de los sesenta" experimentada en casi todo el continente y por el clima de relativa expectación que acompañó a la presencia del contraalmirante Sarmento Rodrigues como gobernador general de of Pancho Guedes, the Dori and Amâncio Guedes Collection, op. cit., p. 61. 
Mozambique en el periodo entre 1961 y 1964 hasta su destitución por Salazar), tuvo dos pilares fundamentales que pueden acreditarse documentalmente: por una parte la reputación internacional que consiguió Amâncio Guedes a partir de 1961, especialmente en Europa, en cuanto arquitecto con obra realizada en Mozambique y por su presencia como mecenas del nuevo arte africano en las principales áreas en las que se demandaba, planificaba y difundía este tipo de arte; y por otra parte la repentina proyección internacional de Malangatana como pintor, lo que conllevaba, además del reconocimiento de su obra, ensalzarlo como un ejemplo de la viabilidad y el éxito inmediato de un nuevo arte africano vinculado a unas raíces locales y un entorno cultural específicos. Para Ulli Beier, "quizá Malangatana haya sido el primer artista africano en encontrar el atajo, en llegar a ser un artista sofisticado sorteando la formación académica" propia del modelo europeo ${ }^{40}$.

En el campo de la arquitectura y con la llegada de los cincuenta, Pancho construyó importantes edificios en Lourenço Marques con un estilo propio (stiloguedes), como los edificios de viviendas Prometheus (1951-1953) y O Leão Que Ri (1954-1955); la Casa Avião (Casa Avión, 1951), la Casa das Três Girafas (Casa de las Tres Jirafas), las Casas Gémeas (Casas Gemelas), las viviendas pareadas Matos Ribeiro, la panadería Saipal y el garaje para Otto Barbosa (1952), el restaurante Zambi (1955), la Escuela Infantil Piramidal (1959-1961) y el hotel de São Martinho de Bilene, proyecto no construido que data de 1951.

Como ya se ha mencionado, la proyección internacional de Pancho lo llevó en abril de 1961 hasta la portada de la revista más influyente de la profesión, Architectural Review, que reproducía un dibujo del hotel diseñado para Bilene. Poco después se publicó en The Times un artículo de J. M. Richards: "Emergence of a new and original figure: remarkable work by Amâncio Guedes"41 (El auge de una personalidad nueva y original: la sorprendente obra de Amâncio Guedes).La búsqueda de un arte popular, y especialmente de pinturas murales, es un rasgo común a Beier, Guedes y Beinart, como muestra su interés por los murales que fueron producto de la distribución de pinturas para alegrar las chabolas de cañizo en Lourenço Marques ante la visita presidencial en 1956 o la decoración del hotel Chuabo en Quelimane ${ }^{42}$.

Según Beinart, "otra manera de tender puentes para permitir que la fuerza de una tradición popular se combine con nuevas ideas y técnicas y se adapte a ellas puede verse en la obra construida por Amâncio Guedes con mozambiqueños que no contaban con formación específica. Guedes, atraído por la habilidad espontánea de aquellas gentes, lleva a cabo una práctica profesional en la que emplea únicamente personal aleccionado por él mismo. En su patio trasero trabaja un ebanista a quien Guedes conoció en una obra, un albañil que realiza murales, el pintor Malangatana, otros colaboradores que dibujan y, recientemente, un costurero para los bordados. Guedes cuenta con algunos de ellos para que trabajen con él en sus proyectos, y la cantidad y calidad del resultado no solo dice mucho de la capacidad creativa y motivadora de Guedes, sino que demuestra que este tipo de colaboraciones puede tener éxito y que este sistema de enseñanza de oficios puede ser un método educativo muy válido en África".

A finales de los años sesenta, Ulli Beier afirma en su libro Contemporary Art in Africa (1968) que "en Lourenço Marques, Salisbury y Oshogbo, el encuentro entre los artistas europeos y africanos desencadenó una nueva actividad creativa y nuevas 'escuelas' de pintura, si no movimientos. [...] La fascinante y atractiva ciudad de Lourenço Marques parece el marco idóneo para el auge de nuevos artistas africanos. No solo los edificios de Pancho Guedes sirven de inspiración constante, sino que existe una comunidad africana muy dinámica que pinta los muros de sus barrios de chabolas con fascinantes diseños rebosantes de jovialidad". En el capítulo "Encontrar un atajo: del arte tradicional africano a las formas de expresión modernas", Beier menciona la importancia de los talleres o comunidades de aprendizaje de oficios que "se han fundado para generar deliberadamente un conjunto de circunstancias, un ambiente en el que pueda tener cabida ese desarrollo". Y añade: "Los de mayor éxito eran el de Pancho Guedes en Lourenço Marques y el de Frank McEwen en Salisbury". Poco después describe el compromiso de Pancho con las actividades de los artistas locales: "Visitar su casa es como visitar el taller de un artista: pintores, escultores y bordadores se distribuyen por todo el espacio. Guedes no imparte clases formales, pero los anima, les critica, les compra sus obras y en ocasiones les proporciona una asignación mensual que permite a los artistas trabajar a tiempo completo sin preocupaciones económicas"³.

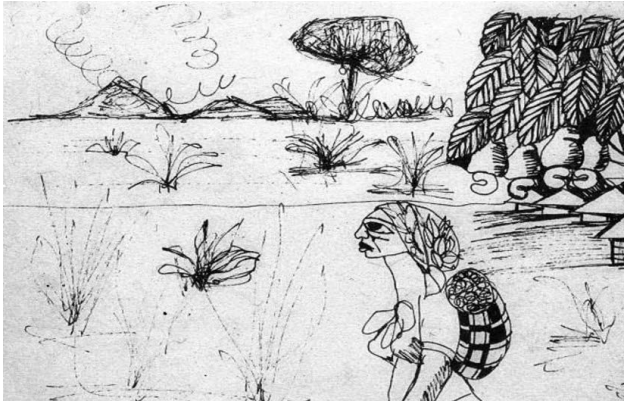

Fig. 5. Dibujo de Malangatana, tinta china, $24 \times 36 \mathrm{~mm}$. () Malangatana Valente Ngwenya Archive, Fundação Mário Soares (http://casacomum.org/cc/visualizador?pasta=07830.003.001).

40. BEIER Ulli, Contemporary Art in Africa, Pall Mall, Londres, 1968, p. 72.

41. Véase nota 22.

42. Proyecto de Arménio Losa y Cassiano Barbosa, junto al arquitecto local Figueirinhas (1955-1962). TOSTÕES, Ana y OLIVEIRA, Maria Manuel, "Transcontinental Modernism. $M \& G$ as an Unité d'habitation and a factory complex in Mozambique", en Docomomo Journal, n. 43, 2010, pp. 70-73. DIAS, Jorge y DIAS, Margot, en A Arte Popular em Portugal, Ilhas Adjacentes e Ultramar, Verbo, Lisboa, 1975, pp. 153161. POMAR, Alexandre (ed.), The Africas of Pancho Guedes, the Dori and Amâncio Guedes Collection, op. cit., pp. 53, 194.

43. POMAR, Alexandre (ed.), The Africas of Pancho Guedes, the Dori and Amâncio Guedes Collection, op. cit., pp. 53. 


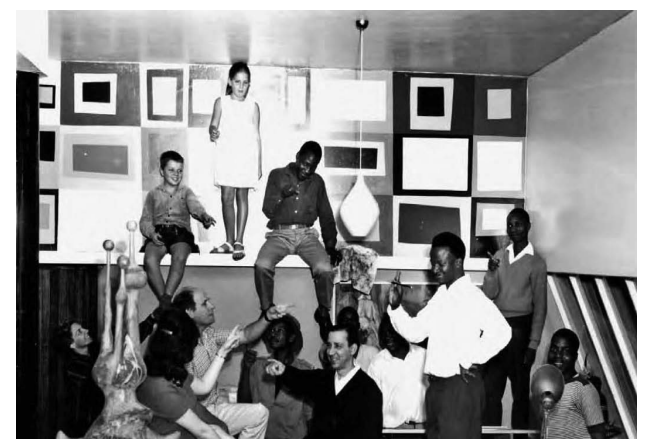

Fig. 6.. En su oficina de Rua de Nevala, Lourenço Marques (Maputo), 1960. Pancho Guedes con su familia, colaboradores y Malangatana (archivo de P. G.).

44. Idem, p. 54.

45. Abitare, n. 501, abril de 2010

46. Idem.

47. GADANHO, Pedro (ed.), Pancho Guedes. Ein alternativer Modernist, Christoph Merian Verlag, Basilea, 2007.

48. TOSTÕES, Ana, "Pancho Guedes, the architect", en POMAR, Alexandre (ed.), The Africas of Pancho Guedes, the Dori and Amâncio Guedes Collection, op. cit., p. 68.
Pomar considera que, junto a la prioridad que concedió a su trabajo como arquitecto, escultor y pintor, Pancho Guedes se interesó por un gran número de artistas africanos o residentes temporalmente en África, ya fueran blancos o negros, cuya obra procuró auspiciar y coleccionar. Invirtió sus esfuerzos en labores que poco tenían que ver con el ideal de una carrera en el mundo de las artes o con los movimientos predominantes, y se decantó por una producción original e idiosincrática que trataba de descubrir artistas populares y amateurs, pues él mismo se consideraba un artista aficionado ${ }^{44}$.

Peter Rich, antiguo estudiante y actualmente también profesor de Witwatersrand, afirma que "la obra del arquitecto Pancho Guedes es una fusión del movimiento moderno europeo con la sensibilidad artística africana"45. Según él "Pancho era un gran mentor porque él mismo era pintor, escultor, dadaísta, surrealista y contagiaba su inagotable energía. Fuimos muy afortunados en la universidad; cualquier persona, tanto los estudiantes como el personal, veía aumentar su potencial en un $300 \%$. Creías que podías hacer cualquier cosa, y cuanto más hicieras, mejor eras. Podías rodar una película, esculpir, pintar, actuar en una obra de teatro e igualmente proyectar arquitectura" ${ }^{346}$.

Desde aspectos técnicos a enfoques poéticos, pasando por el pop art y las manifestaciones del espíritu africano, Pancho defendió una modernidad derivada de un complejo procedimiento que se nutría de fuentes culturales diversas y poco convencionales. Logró esquivar la hegemonía colonial de su época y se sumergió en una miríada de influencias y elementos culturales que dieron forma a un cosmopolitismo africano muy particular en la ciudad de Lourenço Marques durante los años cincuenta y comienzos de los sesenta ${ }^{47}$. Al mismo tiempo, supo capitanear con audacia un nuevo tipo de mecenazgo que daría pie al nacimiento de un nuevo arte africano íntimamente vinculado al carácter de sus raíces locales y sus condicionantes culturales, y luchó por que los artistas tuvieran una oportunidad. Pancho reivindicó para los arquitectos "los derechos y libertades de los que han disfrutado pintores y poetas desde hace tanto tiempo". En cuanto a su propia obra arquitectónica, desarrolló un estilo original de gran capacidad comunicativa cuyas influencias abarcan desde las pinturas de Picasso a las de su amigo Malangatana, con el que compartió investigaciones y creatividad desde un onírico universo freudiano hasta la escultura africana, todo ello mezclado con una expresividad predispuesta al dadaísmo fruto de su amistad con Tristan Tzara ${ }^{48}$.

Gracias a sus vínculos con la población local, descubrió en África una atmósfera favorable para la realización de sus proyectos. Su interés por temas que se extienden desde la escultura africana o la arquitectura indígena hasta Malangatana y desde Gaudí al art nouveau pone de manifiesto los fundamentos de su obra: abierta y heterodoxa, ecléctica e irreverente; una obra que reflexiona en libertad y reinventa en sus construcciones tanto el arte primitivo como el moderno, y cuyos proyectos arquitectónicos se nutren de la pintura y la escultura en un proceso creativo global. Malangatana reconocía que "ningún arquitecto había vinculado antes la arquitectura con la cultura indígena. En sus diseños podemos distinguir una geometría que refleja patrones similares a los tatuajes de la mitología africana". Famoso por su fértil imaginación, para él cada proyecto brota con naturalidad del entorno, del clima, de la geología y de la cultura de aquellos que lo habitan.

Como demuestra su prolífico legado, Guedes anticipó diferentes tendencias y líneas de pensamiento que todavía están por explorar en el contexto internacional y que tienen mucho que aportar a la relación entre arte y arquitectura.

Ana Tostões. Es Arquitecta, doctora en Crítica e Historia de la Arquitectura. Presidenta del Docomomo Internacional y editora del Docomomo Journal. Es profesora en la Universidad de Lisboa, y directora de sus programas de doctorado. Ha ejercido como docente en universidades de todo el mundo. Graduada en Arquitectura (ESBAL, 1982), Máster en Historia del arte (UNL,1994) con la tesis titulada Os Verdes Anos na Arquitectura Portuguesa dos Anos 50 (FAUP Ed., 1997). Doctora en cultura y tecnología en la Arquitectura Moderna con la tesis Idade Maior (FAUP Ed., 2015), con la que fue galardonada en los premios de la X Bienal Iberoamericana de Aquitectura y Urbanismo de 2016. Ha coordinado el proyecto de investigación Exchanging World Visions (PTDC/AUR-AQI/103229/2008) centrado en la arquitectura desarrollada en el áfrica sub-sahariana durante el movimiento moderno, que fue publicado y premiado con el premio Gulbenkian en 2014. Actualmente coordina el Proyecto de investigación Cure and Care: the rehabilitation (PTDC/ATP-AQI/2577/2014). En 2006, el Excelentísmo Presidente de la República Portuguesa la nombró Comadante de la Orden del Infante Dom Henrique por su contribución a la promoción de la arquitectura portuguesa en Portugal y el extranjero. 